\title{
A Computationally Efficient Elastic Wave Model for Media with Power-Law Absorption
}

\author{
Bradley E. Treeby* and B. T. Cox \\ Department of Medical Physics and Bioengineering, University College London, UK \\ *Email: b.treeby@ucl.ac.uk
}

\begin{abstract}
The absorption of ultrasound waves in biological tissue has been experimentally shown to follow a frequency power law. This type of behaviour can be modelled using fractional derivative operators. However, previous elastic wave equations are based on fractional derivatives that are nonlocal in time. This makes them difficult to solve using standard numerical techniques in a memory efficient manner. Here, a fractional Kelvin-Voigt model is derived based on the fractional Laplacian. This is obtained by splitting the particle velocity into compressional and shear components using a dyadic wavenumber tensor. This allows the temporal derivatives to be replaced with spatial derivatives using the lossless dispersion relation with the appropriate compressional or shear wave speed. If the spatial gradients are computed using the Fourier collocation spectral method, this results in a computationally efficient elastic wave model that can account for arbitrary power law absorption of both compressional and shear waves.
\end{abstract}

\section{INTRODUCTION}

Accurately accounting for the absorption of ultrasound waves in biological tissue is of critical importance in many modelling problems, including treatment planning for therapeutic ultrasound [1], and image reconstruction in photoacoustic tomography [2]. Experimentally, acoustic absorption in both soft tissue and bones has been observed to follow a frequency power law of the form $\alpha_{0} \omega^{y}$, where $\omega$ is the temporal frequency and $y$ is between 0 and 2. This type of behaviour can be modelled through the use of fractional derivative operators [3]. However, previous elastic constitutive equations including power law absorption are based on temporal fractional derivatives. Because these operators are non-local in time, numerically they require the time history of the field variables to be stored. This carries a significant computational penalty, particularly in the elastic case where the field variables are vectors and tensors. Here, we show how this penalty can be overcome through the use of absorption operators that are nonlocal in space, rather than time. If the Fourier spectral method is used to compute spatial gradients, this allows different power law absorption parameters for compressional and shear waves to be modelled in a computationally efficient manner.

\section{Model DeVElopment}

\section{A. Kelvin-Voigt Model}

In an elastic material, the strain at each point within a body is only dependent on the instantaneous local stress. The stress and strain are related by the stiffness, which is a measure of resistance to deformation in response to an applied force. For an anisotropic medium, this relationship can be written using Einstein summation notation as

$$
\sigma_{i j}=C_{i j k l} \varepsilon_{k l}
$$

where $\sigma$ is the stress tensor, $\varepsilon$ is the strain tensor, and $C$ is the stiffness tensor. For small deformations, the relationship between strain and particle displacement $u$ is given by

$$
\varepsilon_{i j}=\frac{1}{2}\left(\frac{\partial u_{i}}{\partial x_{j}}+\frac{\partial u_{j}}{\partial x_{i}}\right) .
$$

These expressions describe purely elastic deformations that do not dissipate energy when a stress is applied and removed, i.e., they are lossless equations. Conversely, many materials (including biological tissue) exhibit a time-dependent strain when a stress is applied.

To account for viscoelastic behaviour, Eq. (1) can be generalised by adding additional terms proportional to derivatives of the stress and strain. The most common models are the Maxwell, Kelvin-Voigt, and Zener models [4]. These can each be described by equivalent mechanical circuits, where elastic springs and viscous dampers are cascaded together in different fashions. The Kelvin-Voigt model (which consists of a spring and damper in parallel) is given by

$$
\sigma_{i j}=C_{i j k l} \varepsilon_{k l}+N_{i j k l} \frac{\partial}{\partial t} \varepsilon_{k l},
$$

where $N$ is the viscosity tensor. If the medium is isotropic, the relationship between the stress and strain does not depend on the orientation of the material. In this case, there are only two independent components of the stiffness and viscosity tensors. The Kelvin-Voigt stress-strain relation can then be written in the form

$$
\sigma_{i j}=\lambda \delta_{i j} \varepsilon_{k k}+2 \mu \varepsilon_{i j}+\chi \delta_{i j} \frac{\partial}{\partial t} \varepsilon_{k k}+2 \eta \frac{\partial}{\partial t} \varepsilon_{i j} .
$$

Here $\lambda$ and $\mu$ are the Lamè parameters where $\mu$ is the shear modulus (the ratio of shear stress and shear strain), and $\chi$ and $\eta$ are the compressional and shear viscosity coefficients. The Lamè parameters are related to the shear (transverse) and compressional (longitudinal) sound speeds by

$$
\mu=c_{s}^{2} \rho_{0}, \quad \lambda+2 \mu=c_{p}^{2} \rho_{0} .
$$

where $\rho_{0}$ is the mass density.

To model the propagation of elastic waves, it is necessary to combine the appropriate stress-strain relation with Newton's second law. This is a statement of the conservation of momentum (sometimes referred to as the equation of motion). Written as a function of stress and particle velocity, where $v_{i}=\partial u_{i} / \partial t$, this is given by

$$
\frac{\partial v_{i}}{\partial t}=\frac{1}{\rho_{0}} \frac{\partial \sigma_{i j}}{\partial x_{j}}
$$


The Kelvin-Voigt stress-strain relation can similarly be written as a function of the stress and particle velocity using Eq. (2)

$$
\begin{aligned}
\frac{\partial \sigma_{i j}}{\partial t}= & \lambda \delta_{i j} \frac{\partial v_{k}}{\partial x_{k}}+\mu\left(\frac{\partial v_{i}}{\partial x_{j}}+\frac{\partial v_{j}}{\partial x_{i}}\right) \\
& +\chi \delta_{i j} \frac{\partial^{2} v_{k}}{\partial x_{k} \partial t}+\eta\left(\frac{\partial^{2} v_{i}}{\partial x_{j} \partial t}+\frac{\partial^{2} v_{j}}{\partial x_{i} \partial t}\right) .
\end{aligned}
$$

Equations (6) and (7) are coupled equations that describe the propagation of compressional and shear waves in a viscoelastic solid. These can also be combined into a single elastic wave equation. Written as a function of the particle displacement $u$, this becomes [4, p. 207]

$$
\begin{aligned}
\rho_{0} \frac{\partial^{2} u_{i}}{\partial t^{2}}= & (\lambda+\mu) \frac{\partial^{2}}{\partial x_{i} \partial x_{j}} u_{j}+\mu \frac{\partial^{2}}{\partial x_{j}^{2}} u_{i} \\
& +(\chi+\eta) \frac{\partial^{2}}{\partial x_{i} \partial x_{j}} \frac{\partial u_{j}}{\partial t}+\eta \frac{\partial^{2}}{\partial x_{j}^{2}} \frac{\partial u_{i}}{\partial t} .
\end{aligned}
$$

Using vector notation, this is equivalent to

$$
\begin{aligned}
\rho_{0} \frac{\partial^{2} \mathbf{u}}{\partial t^{2}}= & (\lambda+\mu) \nabla(\nabla \cdot \mathbf{u})+\mu \nabla^{2} \mathbf{u} \\
& +(\chi+\eta) \nabla\left(\nabla \cdot \frac{\partial \mathbf{u}}{\partial t}\right)+\eta \nabla^{2} \frac{\partial \mathbf{u}}{\partial t}
\end{aligned}
$$

Expanding the vector Laplacian using $\nabla^{2} \mathbf{u}=\nabla(\nabla \cdot \mathbf{u})-$ $\nabla \times(\nabla \times \mathbf{u})$ and replacing the Lamè parameters with the compressional and shear sound speeds then gives

$$
\begin{aligned}
\frac{\partial^{2} \mathbf{u}}{\partial t^{2}}= & c_{p}^{2} \nabla(\nabla \cdot \mathbf{u})-c_{s}^{2} \nabla \times(\nabla \times \mathbf{u}) \\
& +\tau_{p} \nabla\left(\nabla \cdot \frac{\partial \mathbf{u}}{\partial t}\right)-\tau_{s} \nabla \times\left(\nabla \times \frac{\partial \mathbf{u}}{\partial t}\right),
\end{aligned}
$$

where $\tau_{p}=\frac{\chi+2 \eta}{\rho_{0}}$ and $\tau_{s}=\frac{\eta}{\rho_{0}}$. This expression can also be written in terms of scalar and vector potentials, where $\mathbf{u}=\nabla \phi+\nabla \times \boldsymbol{\Psi}$ (this is sometimes called the Helmholtz decomposition)

$$
\begin{aligned}
0= & \nabla\left(\frac{\partial^{2} \phi}{\partial t^{2}}-c_{p}^{2} \nabla^{2} \phi-\tau_{p} \frac{\partial}{\partial t} \nabla^{2} \phi\right) \\
& +\nabla \times\left(\frac{\partial^{2} \boldsymbol{\Psi}}{\partial t^{2}}-c_{s}^{2} \nabla^{2} \boldsymbol{\Psi}-\tau_{s} \frac{\partial}{\partial t} \nabla^{2} \boldsymbol{\Psi}\right) .
\end{aligned}
$$

Individual equations for the potentials can then be obtained by taking the divergence or curl of Eq. (11), where $\nabla \cdot \mathbf{u}=\nabla^{2} \phi$ and $\nabla \times \mathbf{u}=\nabla^{2} \boldsymbol{\Psi}$. This results in two separate wave equations given by the bracketed terms in Eq. (11). Each of these is in the form of Stokes' classical viscous wave equation. The parameter $\tau$ can thus be interpreted as Stokes' relaxation time. For $\omega \tau \ll 1$, the acoustic absorption varies with the square of frequency while the sound speed is approximately constant (meaning there is no dispersion). Similarly, for $\omega \tau \gg 1$, both the absorption and the sound speed vary with the square root of frequency [5].

\section{B. Fractional Kelvin-Voigt Model}

In the low frequency limit, the Kelvin-Voigt model described in the previous section accounts for acoustic absorption that varies with frequency squared. However, the absorption that is experimentally observed in biological tissue varies with a power law dependence that can be between 0 and 2 , and is often close to 1. To account for this behaviour, the Maxwell, Kelvin-Voigt, and Zener models can each be generalised by replacing the $\partial / \partial t$ terms with fractional derivatives of the form $\partial^{y} / \partial t^{y}$ (see [3] for a recent review of fractional governing equations). For Eq. (3), this leads to the fractional KelvinVoigt model [6], [7]

$$
\sigma_{i j}=C_{i j k l} \varepsilon_{k l}+N_{i j k l} \frac{\partial^{y-1}}{\partial t^{y-1}} \varepsilon_{k l} .
$$

In the isotropic case, this can be written in the form

$$
\sigma_{i j}=\lambda \delta_{i j} \varepsilon_{k k}+2 \mu \varepsilon_{i j}+\chi \delta_{i j} \frac{\partial^{y-1}}{\partial t^{y-1}} \varepsilon_{k k}+2 \eta \frac{\partial^{y-1}}{\partial t^{y-1}} \varepsilon_{i j} .
$$

Following the same steps as before, this leads to the following wave equation written in terms of scalar and vector potentials

$$
\begin{aligned}
0= & \nabla\left(\frac{\partial^{2} \phi}{\partial t^{2}}-c_{p}^{2} \nabla^{2} \phi-\tau_{p} \frac{\partial^{y-1}}{\partial t^{y-1}} \nabla^{2} \phi\right) \\
& +\nabla \times\left(\frac{\partial^{2} \boldsymbol{\Psi}}{\partial t^{2}}-c_{s}^{2} \nabla^{2} \boldsymbol{\Psi}-\tau_{s} \frac{\partial^{y-1}}{\partial t^{y-1}} \nabla^{2} \boldsymbol{\Psi}\right) .
\end{aligned}
$$

In this case, the equations for the individual potentials are now both in the form of the Caputo/Wismer fractional waveequation [7], [8]. Written in terms of the acoustic pressure $p$, this is given by

$$
\frac{1}{c_{0}^{2}} \frac{\partial^{2}}{\partial t^{2}} p-\nabla^{2} p-\tau \frac{\partial^{y-1}}{\partial t^{y-1}} \nabla^{2} p=0
$$

In the low frequency limit where $\omega \tau \ll 1$, this equation encapsulates power-law acoustic absorption of the form

$$
\alpha=-\frac{\tau \cos (\pi y / 2)}{2 c_{0}} \omega^{y},
$$

where $\alpha$ is the absorption coefficient in $\mathrm{Npm}^{-1}$. For the elastic case with $\omega \tau_{p} \ll 1$ and $\omega \tau_{s} \ll 1$, the absorption of compressional and shear waves is thus governed by

$$
\alpha_{p}=-\frac{(\chi+2 \eta) \cos (\pi y / 2)}{2 \rho_{0} c_{p}^{3}} \omega^{y}, \quad \alpha_{s}=-\frac{\eta \cos (\pi y / 2)}{2 \rho_{0} c_{s}^{3}} \omega^{y} .
$$

To account for absorption of the form $\alpha=\alpha_{0} \omega^{y}$, this means the viscosity coefficients should be chosen such that

$$
\eta=-\frac{2 \rho_{0} c_{s}^{3}}{\cos (\pi y / 2)} \alpha_{0, s}, \quad \chi=-\frac{2 \rho_{0} c_{p}^{3}}{\cos (\pi y / 2)} \alpha_{0, p}-2 \eta,
$$

where $\alpha_{0, s}$ and $\alpha_{0, p}$ are the desired absorption coefficient pre-factors for shear and compressional waves in $\mathrm{Np}(\mathrm{rad} / \mathrm{s})^{-y} \mathrm{~m}^{-1}$, and $y$ is the desired power law dependence.

\section{From Temporal to Spatial Fractional Derivatives}

The use of fractional time derivatives in the Kelvin-Voigt model discussed in the previous section introduces an interesting computational problem. This arises because the fractional operators are non-local in time. To illustrate, the fractional derivative in Caputo's form may be written as the convolution between an integer derivative and a function $\Phi$

$$
\frac{\partial^{y} f(t)}{\partial t^{y}}=\frac{\partial^{n} f(t)}{\partial t^{n}} * \Phi_{n-y}(t)
$$




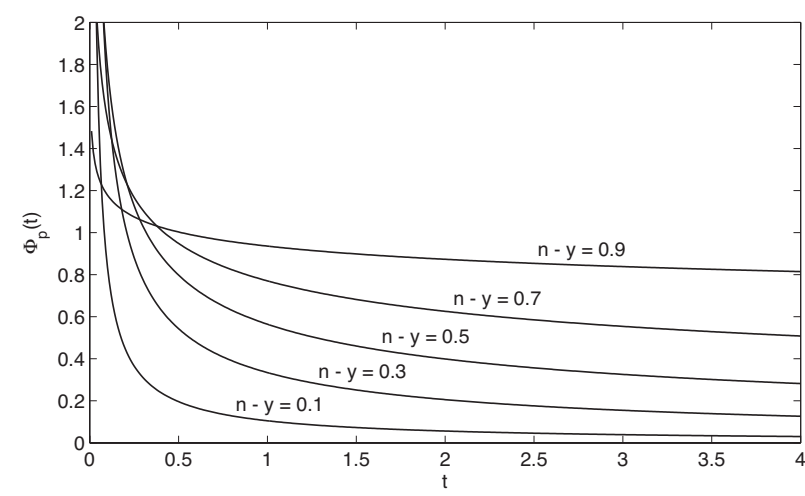

Fig. 1. Decay of the convolution function $\Phi_{n-y}(t)$ used in Caputo's fractional derivative for different values of $n-y$.

where

$$
\Phi_{n-y}(t)=\frac{t^{n-y-1}}{\Gamma(n-y)} \quad \text { for } \quad t>0 .
$$

Here $y$ is a positive real number (which may be fractional), $n$ is the next integer value equal to or larger than $y$, and * denotes a convolution. As shown in Fig. 1, the function $\Phi_{n-y}(t)$ has a long tail that decays more and more slowly as $n-y$ moves from 0 to 1 . For the fractional Kelvin-Voigt model given in Eq. (13), the fractional power is $y-1$. This means if the power law exponent $y$ is close to 1 (as often observed in biological tissue), the function $\Phi_{n-y}(t)$ will have a very long tail. Practically, this means to evaluate the fractional derivative using the convolution in Eq. (19), a large time history of $f(t)$ must be stored. For explicit time-stepping methods, this can carry a large computational penalty. For example, Wismer noted that for $y=1.4$, even storing the previous 20 time steps was not sufficient to accurately evaluate the fractional derivative [8].

Under certain conditions, it is possible to replace fractional time derivatives with fractional space derivatives which are non-local in space, rather than time [9]. For explicit timestepping methods, this has a significant computational benefit, as the wave-field at other spatial positions is already known. To illustrate how this replacement arises, consider the spatial and temporal Fourier transform of the fractional temporal derivative of a function $f(x, t)$

$$
\mathcal{F}\left\{\frac{\partial^{y} f(x, t)}{\partial t^{y}}\right\}=(-i \omega)^{y} F(k, \omega) .
$$

Here $k$ and $\omega$ are the spatial and temporal frequencies, and $\mathcal{F}\{\ldots\}$ denotes the Fourier transform. The first part of this expression can be expanded using $(-i)^{y}=\cos (\pi y / 2)-$ $i \sin (\pi y / 2)$ to give

$$
(-i \omega)^{y}=\cos (\pi y / 2) \omega^{y}+(-i \omega) \sin (\pi y / 2) \omega^{y-1} .
$$

For many applications (including biomedical ultrasound), acoustic absorption only has a second order effect on wave propagation. This means that the temporal frequency terms in Eq. (22) (which correspond to temporal derivatives) can be replaced by spatial frequency terms (which correspond to spatial derivatives) using the dispersion relation for the lossless wave equation $\omega=k c_{0}$. This is based on the premise that the substitution of first-order relations into second-order terms will result in third-order errors. Equation (22) then becomes

$$
(-i \omega)^{y} \approx \cos (\pi y / 2) k^{y} c_{0}^{y}+(-i \omega) \sin (\pi y / 2) k^{y-1} c_{0}^{y-1} .
$$

Using the definition of the fractional Laplacian [10]

$$
\mathcal{F}\left\{\left(-\nabla^{2}\right)^{y} f(x, t)\right\}=k^{2 y} F(k, \omega),
$$

and taking the inverse Fourier transform of Eq. (23) then yields

$$
\begin{aligned}
\frac{\partial^{y}}{\partial t^{y}} \approx c_{0}^{y} & \cos (\pi y / 2)\left(-\nabla^{2}\right)^{y / 2} \\
& +c_{0}^{y-1} \sin (\pi y / 2)\left(-\nabla^{2}\right)^{(y-1) / 2} \frac{\partial}{\partial t} .
\end{aligned}
$$

Thus fractional temporal derivatives can be replaced with fractional spatial derivatives provided the effect of absorption on the wave-field is small. To illustrate, substituting Eq. (25) into Eq. (15) leads to the fractional Laplacian wave equation

$$
\frac{1}{c_{0}^{2}} \frac{\partial^{2} p}{\partial t^{2}}-\nabla^{2} p-\tau_{1}\left(-\nabla^{2}\right)^{y / 2} \frac{\partial}{\partial t} p-\tau_{2}\left(-\nabla^{2}\right)^{(y+1) / 2} p=0
$$

where $\tau_{1}=2 \alpha_{0} c_{0}^{y-1}$ and $\tau_{2}=2 \alpha_{0} c_{0}^{y} \tan (\pi y / 2)$. This is the absorption model derived in Refs. [10], [9] and implemented in the k-Wave toolbox [11]. If the Fourier spectral method is used to compute the spatial gradients, the fractional Laplacian terms become trivial to compute, where

$$
\left(-\nabla^{2}\right)^{y} f(x, t)=\mathcal{F}^{-1}\left\{k^{2 y} \mathcal{F}\{f(x, t)\}\right\} .
$$

In the fluid case, this approach has been shown to be computationally efficient [9]. However, in the elastic case, the compressional and shear waves travel at two different sound speeds. This means the relation $\omega=k c_{0}$ cannot be used with Eq. (22) to convert temporal to spatial fractional derivatives. In the next section, we discuss how this restriction can be overcome by splitting the displacement or velocity fields into shear and compressional components in $k$-space.

\section{Field-Splitting in $k$-space}

Considering first the lossless case and following Ref. [12], the wave equation given in Eq. (10) can be written in the spatial frequency domain as

$$
\frac{\partial^{2} \mathbf{U}}{\partial t^{2}}=c_{p}^{2} i \mathbf{k}(i \mathbf{k} \cdot \mathbf{U})-c_{s}^{2} i \mathbf{k} \times(i \mathbf{k} \times \mathbf{U}),
$$

where $\mathcal{F}_{x}\{\nabla\}=i \mathbf{k}$ and $\mathcal{F}_{x}\{\mathbf{u}\}=\mathbf{U}$. The first term in this expression can be expanded using the substitution $\mathbf{k}(\mathbf{k} \cdot \mathbf{U})=(\mathbf{k k}) \cdot \mathbf{U}$, where $\mathbf{k k}$ is the dyadic tensor formed by the outer product of $\mathbf{k}$ with itself. Similarly, the second term can be expanded using the triple vector product $\mathbf{a} \times(\mathbf{b} \times \mathbf{c})=\mathbf{b}(\mathbf{a} \cdot \mathbf{c})-\mathbf{c}(\mathbf{a} \cdot \mathbf{b})$. This leads to $\mathbf{k} \times(\mathbf{k} \times \mathbf{U})=$ $\left(\mathbf{k k}-k^{2} \mathbf{I}\right) \cdot \mathbf{U}$, where $k^{2} \equiv \mathbf{k} \cdot \mathbf{k}$ and $\mathbf{I}$ is the identity matrix. Using these expansions, Eq. (28) can then be written in the form

$$
\frac{\partial^{2} \mathbf{U}}{\partial t^{2}}=-k^{2}\left(c_{p}^{2}(\hat{\mathbf{k}} \hat{\mathbf{k}})+c_{s}^{2}(\mathbf{I}-\hat{\mathbf{k}} \hat{\mathbf{k}})\right) \cdot \mathbf{U},
$$

where $\hat{\mathbf{k}}=\mathbf{k} / k$ is the normalised wavenumber vector. 
The dyadic operators $(\hat{\mathbf{k}} \hat{\mathbf{k}})$ and $(\mathbf{I}-\hat{\mathbf{k}} \hat{\mathbf{k}})$ in Eq. (29) act to split the vector particle displacement into compressional and shear components, i.e.,

$$
\mathbf{U}^{p}=(\hat{\mathbf{k}} \hat{\mathbf{k}}) \cdot \mathbf{U}, \quad \mathbf{U}^{s}=(\mathbf{I}-\hat{\mathbf{k}} \hat{\mathbf{k}}) \cdot \mathbf{U},
$$

where $\mathbf{U}=\mathbf{U}^{p}+\mathbf{U}^{s}$. Written using Einstein summation notation this is equivalent to

$$
U_{i}^{p}=\hat{k}_{i} \hat{k}_{j} U_{j}, \quad U_{i}^{s}=\left(\delta_{i j}-\hat{k}_{i} \hat{k}_{j}\right) U_{j} .
$$

Using the same approach to split the particle velocity, the fractional Kelvin-Voigt stress-strain relation can be split into two equations which separately describe the compressional and shear components of the wave-field. Written in $x$ - $t$ space, this gives

$$
\begin{aligned}
\frac{\partial \sigma_{i j}^{p, s}}{\partial t}= & \lambda \delta_{i j} \frac{\partial v_{k}^{p, s}}{\partial x_{k}}+\mu\left(\frac{\partial v_{i}^{p, s}}{\partial x_{j}}+\frac{\partial v_{j}^{p, s}}{\partial x_{i}}\right) \\
& +\chi \delta_{i j} \frac{\partial}{\partial x_{k}} \frac{\partial^{y-1}}{\partial t^{y-1}} v_{k}^{p, s} \\
& +\eta\left(\frac{\partial}{\partial x_{j}} \frac{\partial^{y-1}}{\partial t^{y-1}} v_{i}^{p, s}+\frac{\partial}{\partial x_{i}} \frac{\partial^{y-1}}{\partial t^{y-1}} v_{j}^{p, s}\right),
\end{aligned}
$$

where the total stress field is $\sigma_{i j}=\sigma_{i j}^{p}+\sigma_{i j}^{s}$. Because the field is split, the fractional temporal derivatives can now be replaced with fractional spatial derivatives using Eq. (25), where $c_{0}$ is chosen to be the appropriate shear or compressional sound speed. This also allows the absorption power law exponent $y$ to be chosen separately for the shear and compressional waves to match experimental data.

\section{E. Pseudospectral Time Domain Solution}

A computationally efficient model for elastic wave propagation in absorbing media can now be constructed using the split-field fractional Kelvin-Voigt model along with the equation of motion given in Eq. (6). Here, these are solved as two coupled partial differential equations using the Fourier collocation spectral method to compute spatial gradients. This leads to

$$
\begin{aligned}
\frac{\partial \sigma_{i j}^{p}}{\partial t} & =\lambda \mathcal{F}^{-1}\left\{\delta_{i j} k_{k} V_{k}^{p}\right\}+\mu \mathcal{F}^{-1}\left\{i k_{j} V_{i}^{p}+i k_{i} V_{j}^{p}\right\} \\
& +\chi \mathcal{F}^{-1}\left\{\delta_{i j} k_{k} L_{p} V_{k}^{p}\right\}+\eta \mathcal{F}^{-1}\left\{i k_{j} L_{p} V_{i}^{p}+i k_{i} L_{p} V_{j}^{p}\right\} \\
\frac{\partial \sigma_{i j}^{s}}{\partial t} & =\mu \mathcal{F}^{-1}\left\{i k_{j} V_{i}^{s}+i k_{i} V_{j}^{s}\right\} \\
& +\eta \mathcal{F}^{-1}\left\{i k_{j} L_{s} V_{i}^{s}+i k_{i} L_{s} V_{j}^{s}\right\},
\end{aligned}
$$

where the split velocity components are calculated from

$$
V_{j}=\mathcal{F}\left\{v_{j}\right\}, \quad V_{i}^{p}=\hat{k}_{i} \hat{k}_{j} V_{j}, \quad V_{i}^{s}=\left(\delta_{i j}-\hat{k}_{i} \hat{k}_{j}\right) V_{j},
$$

and the parameters $L_{p}$ and $L_{s}$ are given by

$$
L_{p, s}=k^{y-1} c_{p, s}^{y-1} \sin (\pi y / 2)-k^{y-2} c_{p, s}^{y-2} \cos (\pi y / 2) \frac{\partial}{\partial t} .
$$

This is coupled with the equation of motion from Eq. (6), which can be written in the form

$$
\frac{\partial v_{i}}{\partial t}=\frac{1}{\rho_{0}} \mathcal{F}^{-1}\left\{i k_{j} \mathcal{F}\left\{\sigma_{i j}\right\}\right\},
$$

where the split components of the stress field are combined before calculating the updates for the particle velocity. The time derivatives in Eqs. (33) and (35) can be discretised using any standard explicit finite-difference scheme [12], [13]. The encapsulated power law absorption is again given by Eq. (17). However, in this case, the power law exponent $y$ can also be chosen separately for the shear and compressional wave components.

\section{CONCLUSION}

A computationally efficient elastic wave model that accounts for the power law absorption of both compressional and shear waves is derived. This is based on a fractional Kelvin-Voigt model which is split into compressional and shear components using a dyadic wavenumber tensor. This allows the temporal fractional derivatives to be replaced with spatial fractional derivatives using the lossless dispersion relation with the appropriate compressional or shear wave speed. If the spatial gradients are computed using the Fourier collocation spectral method, this results in a computationally efficient elastic wave model that can account for the power law absorption of both compressional and shear waves.

\section{ACKNOWLEDGMENT}

This work was supported by the Dosimetry for Ultrasound Therapy project which is part of the European Metrology Research Programme (EMRP). The EMRP is jointly funded by the EMRP participating countries within EURAMET and the European Union.

\section{REFERENCES}

[1] C. W. Connor and K. Hynynen, "Patterns of thermal deposition in the skull during transcranial focused ultrasound surgery." IEEE Trans. Biomed. Eng., vol. 51, no. 10, pp. 1693-706, 2004.

[2] B. E. Treeby, E. Z. Zhang, and B. T. Cox, "Photoacoustic tomography in absorbing acoustic media using time reversal," Inverse Probl., vol. 26, no. 11, p. 115003,2010

[3] S. Holm and S. Näsholm, "Comparison of fractional wave equations for power law attenuation in ultrasound and elastography," arXiv preprint arXiv:1306.6507, 2013.

[4] H. F. Pollard, Sound Waves in Solids. London: Pion Limited, 1977.

[5] J. J. Markham, R. T. Beyer, and R. B. Lindsay, "Absorption of sound in fluids," Reviews of Modern Physics, vol. 23, no. 4, p. 353, 1951.

[6] L. Knopoff, "On the dissipative viscoelastic constants of higher order," J. Acoust. Soc. Am., vol. 26, no. 2, pp. 183-186, 1954.

[7] M. Caputo, "Linear models of dissipation whose Q is almost frequency independent-II," Geophys. J. R. Astr. Soc., vol. 13, pp. 529-539, 1967.

[8] M. G. Wismer, "Finite element analysis of broadband acoustic pulses through inhomogenous media with power law attenuation," J. Acoust. Soc. Am., vol. 120, no. 6, p. 3493, 2006.

[9] B. E. Treeby and B. T. Cox, "Modeling power law absorption and dispersion for acoustic propagation using the fractional Laplacian," $J$. Acoust. Soc. Am., vol. 127, no. 5, pp. 2741-2748, 2010.

[10] W. Chen and S. Holm, "Fractional Laplacian time-space models for linear and nonlinear lossy media exhibiting arbitrary frequency powerlaw dependency," J. Acoust. Soc. Am., vol. 115, no. 4, pp. 1424-1430, 2004

[11] B. E. Treeby and B. T. Cox, "k-Wave: MATLAB toolbox for the simulation and reconstruction of photoacoustic wave fields," J. Biomed. Opt., vol. 15, no. 2, p. 021314, 2010.

[12] K. Firouzi, B. T. Cox, B. E. Treeby, and N. Saffari, "A first-order kspace model for elastic wave propagation in heterogeneous media," $J$. Acoust. Soc. Am., vol. 132, no. 3, pp. 1271-1283, 2012.

[13] B. Fornberg, A Practical Guide to Pseudospectral Methods. Cambridge: Cambridge University Press, 1996. 